\title{
Condición muscular y estabilidad del tronco en judocas de nivel nacional e internacional
}

\author{
Casto JUAN-RECIO, David BARBADO, Alejandro LOPEZ-VALENCIANO, Diego LÓPEZ-PLAZA, \\ Carlos MONTERO-CARRETERO \& Francisco J. VERA-GARCIA ${ }^{1}$ \\ Universidad Miguel Hernández de Elche. Centro de Investigación del Deporte (España)
}

Recepción: 08/10/2013; Aceptación: 29/11/2013; Publicación: 02/12/2013.

\section{Resumen}

Antecedentes: Desde un punto de vista teórico, el desarrollo de la capacidad para estabilizar el tronco podría mejorar el rendimiento del judoca a través del desarrollo del control del equilibrio corporal y la optimización de la transmisión de fuerzas desde los miembros inferiores hacia los miembros superiores. Sin embargo, carecemos de evidencias científicas que permitan establecer una relación clara entre la estabilidad del tronco y el rendimiento en judo.

Objetivo: El objetivo principal del estudio fue determinar si la valoración de la estabilidad del tronco y la fuerza y resistencia muscular permitían diferenciar entre judocas de categoría nacional $(n=7)$ e internacional $(n=6)$. Asimismo, se analizó la relación entre la estabilidad del tronco y la fuerza y resistencia de la musculatura implicada en el control de la estabilidad.

Método: Para valorar la estabilidad del tronco se analizó tanto la respuesta del tronco ante cargas súbitas aplicadas mediante un mecanismo de tracción neumática, como el control postural del tronco mediante el paradigma del asiento inestable. Para valorar la fuerza y resistencia muscular se realizó un test de flexión y extensión del tronco en un dinamómetro isocinético.

Resultados/Conclusiones: Los judocas de categoría internacional mostraron menor desplazamiento del CoP en la tarea más compleja sobre el asiento inestable (7.00 \pm 1.19 vs $8.93 \pm 1.45 \mathrm{~mm} ; \mathrm{p}=.025)$ y mayor momento de fuerza relativo de la musculatura extensora $(7.05 \pm 0.87$ vs $5.74 \pm 0.72 \mathrm{Nm} ; \mathrm{p}=.013)$ que los judocas de categoría nacional. Según estos resultados, la condición muscular y la estabilidad del tronco son cualidades a tener en cuenta en la preparación física de los judocas. El análisis correlacional no encontró relación entre las variables analizadas, por lo que la fuerza y resistencia muscular no parecen tener un efecto importante sobre el rendimiento en los test de estabilidad del tronco.

Palabras clave: Estabilidad del raquis; posturografía; acondicionamiento muscular; dinamometría isocinética; rendimiento deportivo.

Muscular condition and trunk stability in judoka of national and international level

\section{Abstract}

Background: It is theorized that the development of the ability to stabilize the trunk may improve the performance of a judoka because it improves body balance control and optimizes force transmission from the lower extremities to the upper limbs. However, there is a lack of scientific evidence to establish a clear relationship between trunk stability and performance in judo.

Aim: The purpose of this study was to determine whether the quantification of trunk stability and muscular strength and endurance allowed differentiation between national level $(n=7)$ and international level judoka $(n=6)$. In addition, the relationship between trunk stability and muscular strength and endurance of the muscles involved in trunk stability control was analyzed.

Method: To assess trunk stability, trunk responses to sudden loads applied by a pneumatic mechanism were

\section{Condição muscular e estabilidade do tronco nos judocas} de nível internacional

\section{Resumo}

Antecedentes: Do ponto de vista teórico, o desenvolvimento da capacidade para estabilizar o tronco pode melhorar o rendimento do judoca através do desenvolvimento do controlo do equilíbrio corporal e a otimização da transmissão de forças dos membros inferiores e dos membros superiores. Contudo, carecem as evidências que permitem estabelecer uma clara relação entre a estabilidade do tronco e o rendimento no judo.

Objetivo: 0 objetivo do estudo foi o determinar se a valorização da estabilidade do tronco e da força e resistência muscular permitiam diferenciar entre judocas de categoria nacional $(\mathrm{n}=7)$ e judocas de categoria internacional $(n=6)$. Assim mesmo, analisou-se a relação entre a estabilidade do tronco e a força e a resistência muscular implicada e o controlo da estabilidade.

Método: Para medir a estabilidade do tronco, analisou-se a resposta do tronco antes das cargas súbitas aplicadas mediante um mecanismo de tração neumática, bem como o

${ }^{1}$ E-mail: fvera@umh.es. Dirección: Centro de Investigación del Deporte, Universidad Miguel Hernández de Elche. Avda. de la Universidad s/n. 03202. Elche (Alicante), España. Http://www.cid-umh.es. 
analyzed, as well as trunk postural control through an unstable sitting paradigm. Muscular strength and endurance were assessed via a flexion and extension trunk test using an isokinetic dynamometer.

Results/Conclusions: International level judokas showed lower CoP displacement in the most complex task in unstable seat $(7.00 \pm 1.19$ vs $8.93 \pm 1.45 \mathrm{~mm}, \mathrm{~T}=$ $.025)$ and higher absolute and relative peak torque in extensor muscles $(7.05 \pm 0.87$ vs $5.74 \pm 0.72 \mathrm{Nm}, \mathrm{T}=$ .013) than national level judoka. According to these results, core stability and trunk muscular condition are important qualities in the physical training of elite judoka. Correlational analysis found no relation between the analyzed variables, thus muscular strength and endurance appear to have a non-significant effect on performance in the trunk stability tests.

Keywords: Spine stability; posturography; muscle conditioning; isokinetic dynamometry; sports performance. controlo postural do tronco mediante o paradigma da posição instável. Para medir a força e a resistência muscular, realizou-se um teste de flexão e extensão do tronco com um dinamómetro isocinético.

Resultados/Conclusões: Os judocas de categoria internacional mostraram menor deslizamento do CoP na tarefa mais complexa sobre a posição instável $(7.00 \pm 1.19$ vs $8.93 \pm 1.45 \mathrm{~mm} ; \mathrm{p}=.025)$ e maior momento de força relativa à musculatura extensora $(7.05 \pm 0.87$ vs $5.74 \pm 0.72$ $\mathrm{Nm}$; $\mathrm{p}=.013$ ) do que os judocas de categoria nacional. Segundo estes resultados, a condição muscular e a estabilidade do tronco são qualidades a ter em conta na preparação física dos judocas. A análise correlacionada não encontrou relação entre as variáveis analisadas, na medida em que a força e a resistência muscular não parecem ter um efeito importante sobre o rendimento nos testes de estabilidade do tronco.

Palavras-chave: Estabilidade do tronco; posturografia; condicionamento muscular; dinamometria isocinética; rendimento desportivo.

\section{1.- Introducción}

Los ejercicios para el desarrollo de la musculatura del tronco son elementos habituales de los programas de entrenamiento deportivo y se utilizan principalmente con el propósito de prevenir lesiones y mejorar el rendimiento de los deportistas (Borghuis et al., 2008; Reed et al., 2012). En particular, en la última década ha incrementado el interés de los entrenadores, preparadores físicos y rehabilitadores por la utilización de ejercicios para la mejora de la estabilidad del tronco, conocida en ámbitos del entrenamiento y la medicina deportiva como estabilidad de la zona central o estabilidad del core (Borghuis et al., 2008; Kibler et al., 2006; Reed et al., 2012). En biomecánica, el concepto de estabilidad del tronco, hace referencia a la capacidad del cuerpo para mantener o recuperar una posición o trayectoria del tronco cuando este es sometido a fuerzas externas o internas (Zazulak et al., 2008). Estudios biomecánicos y epidemiológicos realizados en diferentes poblaciones de deportistas han relacionado el déficit en el control neuromuscular del tronco ante perturbaciones rápidas aplicadas en diferentes direcciones, con lesiones de la columna lumbar (Cholewicki et al., 2002) y de los miembros inferiores (Zazulak et al., 2007). Asimismo, autores como Kibler et al. (2006) han manifestado que el desarrollo de la estabilidad del core puede ayudar a la mejora del rendimiento deportivo, ya que este es el centro de las cadenas cinéticas que participan en numerosas acciones deportivas, facilitando la transmisión de las fuerzas generadas por los miembros inferiores hacia los miembros superiores y viceversa (Borghuis et al., 2008; Kibler et al., 2006).

El control del equilibrio corporal es una característica fundamental del judoca (Perrin et al., 2002; Yoshitomi et al., 2006), el cual debe mantener el equilibrio ante las fuerzas que le aplica el oponente, a la vez que intenta desequilibrarlo a fin de obtener una situación de ventaja. Teniendo en cuenta que el control de la estabilidad del tronco es fundamental para el equilibrio corporal en bipedestación y apoyo monopodal (Burg et al., 2006; Preuss y Fung, 2008; Sung et al., 2010), el desarrollo de esta capacidad podría ser importante para el rendimiento en la lucha en pie (naguewaza).

Varios estudios biomecánicos realizados por nuestro grupo de investigación (Elvira et al., en prensa; López-Valenciano et al., 2013) han encontrado diferencias en la capacidad de estabilización del tronco entre deportistas de diferentes disciplinas deportivas. Así, por ejemplo, el trabajo de López-Valenciano et al. (2013) reveló que judocas de élite mostraban menor desplazamiento angular y mayor rigidez del tronco ante fuerzas externas aplicadas en dirección lateral en comparación con individuos que practicaban otros deportes. Estas diferencias podrían ser fruto de 
una adaptación específica a la práctica del judo, ya que durante acciones de ataque y defensa el judoca tiene que responder continuamente a fuerzas laterales y oblicuas que retan su estabilidad.

Otra de las adaptaciones producidas por el entrenamiento y la competición en judo es el desarrollo de la fuerza y resistencia de los músculos del tronco. Estudios realizados con dinamómetros isocinéticos han encontrado mayores niveles de fuerza de los músculos del tronco en judocas de categoría nacional e internacional en comparación tanto con deportistas entrenados recreacionalmente (Juan-Recio et al., 2013), como con ciclistas de nivel nacional e internacional (Kort y Hendriks, 1992).

Si bien estos resultados parecen indicar que existen adaptaciones específicas en la zona central asociadas a la práctica del judo frente a la práctica de otros deportes, no existen evidencias suficientes que nos permitan conocer en qué medida el desarrollo de estas capacidades está relacionado con un mayor rendimiento en este u otros deportes (Reed et al., 2012).

Ante esta situación, se desarrolló un estudio con judocas de competición de nivel nacional e internacional donde se midió la fuerza y resistencia de los músculos flexores y extensores del tronco mediante dinamometría isocinética, así como la estabilidad del tronco mediante diferentes técnicas biomecánicas. El objetivo principal fue determinar si alguna de las variables referidas permitía diferenciar entre judocas de nivel nacional e internacional. Asimismo, debido a que se desconoce el efecto de la fuerza y de la resistencia de los músculos del tronco sobre la capacidad de estabilización del raquis y la pelvis, se analizó la relación de estas variables en los judocas. Esta información es importante para profundizar en el conocimiento de aquellos factores que modulan la capacidad de estabilización del tronco y su relación con el rendimiento en el judo de competición.

\section{2.- Método}

\section{1.- Participantes}

Un total de 13 judocas de competición participaron en este estudio: 7 judocas de categoría nacional $(22.14 \pm 6.23$ años, $70.97 \pm 3.94 \mathrm{~kg}, 172.31 \pm 2.61 \mathrm{~cm})$ y 6 judocas de categoría internacional $(26.17 \pm 6.67$ años, $69.28 \pm 5.83 \mathrm{~kg}, 169.20 \pm 4.62 \mathrm{~cm})$. Los deportistas eran hombres y fueron reclutados en varios clubes de judo de la costa sur-este de España, gracias a la colaboración de entrenadores locales. Todos los judocas tenían más de 4 años de experiencia en campeonatos de España y los judocas de nivel internacional, además, habían sido seleccionados por el equipo nacional de España para participar en torneos internacionales puntuables para el ranking mundial. No se incluyeron en el estudio judocas con alteraciones raquídeas o dolor lumbar durante el registro de los datos o en los doce meses anteriores al mismo, así como aquellos que habían sido sometidos a cirugía raquídea. Todos los participantes fueron informados de los riesgos del estudio y firmaron un consentimiento informado antes de la investigación (Declaración de Helsinki de 2008). El Comité de Ética de la Universidad dio su aprobación para la realización del estudio.

\section{2.- Procedimiento}

Para valorar la estabilidad del tronco se utilizaron dos metodologías diferentes: i) se analizó la respuesta del tronco ante cargas o perturbaciones unidireccionales aplicadas de forma súbita (sudden load) (Vera-Garcia et al., 2006, 2007); y ii) se evaluó el control postural del tronco mediante el paradigma del asiento inestable (unstable sitting) (Cholewicki et al., 2000; Elvira et al., en prensa; Lee y Granata, 2008; van Dieen et al., 2010a, 2010b). Asimismo, para valorar la fuerza y resistencia de los músculos del tronco se realizó un test de flexión y extensión del tronco en un dinamómetro isocinético Biodex ${ }^{\circledR}$ (Modelo 2000, Sistema Multi-empalme 4 Pro, Biodex Corporation, Shirley, NY, EE.UU.)

Antes de los test, los participantes realizaron un calentamiento que consistió en la realización de 2 series de 15 flexiones del tronco en decúbito supino (crunches o curl-ups) (McGill, 2002) y 2 series de 15 extensiones del tronco en banco romano (back extensions) (McGill, 2002). La recuperación entre series y ejercicios fue de $30 \mathrm{~s}$. 


\subsection{1.- Test de aplicación de cargas súbitas}

Los participantes se colocaron en posición semi-sentada sobre una silla de madera que facilita la colocación del raquis en posición neutra (Sutarno \& McGill, 1995) (Fig. 1). Esta silla limita el movimiento de la pelvis, al mismo tiempo que deja el tronco libre para moverse en todas direcciones. En esta situación, los participantes recibieron cargas súbitas e inesperadas mediante un sistema de tracción formado por un pistón neumático ( 4.2 bares de presión), un tensor y un arnés ajustable colocado sobre el tórax (Fig. 1). El sistema se colocó delante, detrás y a la izquierda de los participantes para aplicar las cargas repentinas en dirección sagital y sentido anterior, en dirección sagital y sentido posterior y en dirección lateral y sentido hacia la izquierda, respectivamente. El sistema es ajustable, lo que permitió que las cargas se aplicaran siempre horizontalmente y sobre el centro de masas de la parte superior del cuerpo de los participantes, conocida como HAT $(0.626$ de la distancia desde el trocánter mayor hasta la articulación acromioclavicular) (Winter, 1990). Se aplicaron cinco cargas súbitas en cada dirección, cada una de ellas sin previo aviso dentro de una ventana de 30 a $45 \mathrm{~s}$ donde los participantes estaban en reposo. Con el fin de evitar la influencia de la fatiga sobre la respuesta del tronco ante las cargas, se dejó 1 min de descanso entre cada dirección analizada.

Para medir la magnitud de la carga aplicada y determinar el inicio de la perturbación se colocó una célula de carga (Transductor Técnicas Inc., Temecula, CA, EE.UU) entre el pistón neumático y el tensor (Fig. 1). Las señales de fuerza fueron amplificadas y convertidas A/D (resolución de 16 bits y rango de entrada de $\pm 5 \mathrm{~V}$ ) a $1000 \mathrm{~Hz}$.

Figura 1. Vista lateral de la silla de madera y del mecanismo de tracción neumática situado frente al sujeto para la aplicación de cargas súbitas en dirección sagital y sentido anterior.

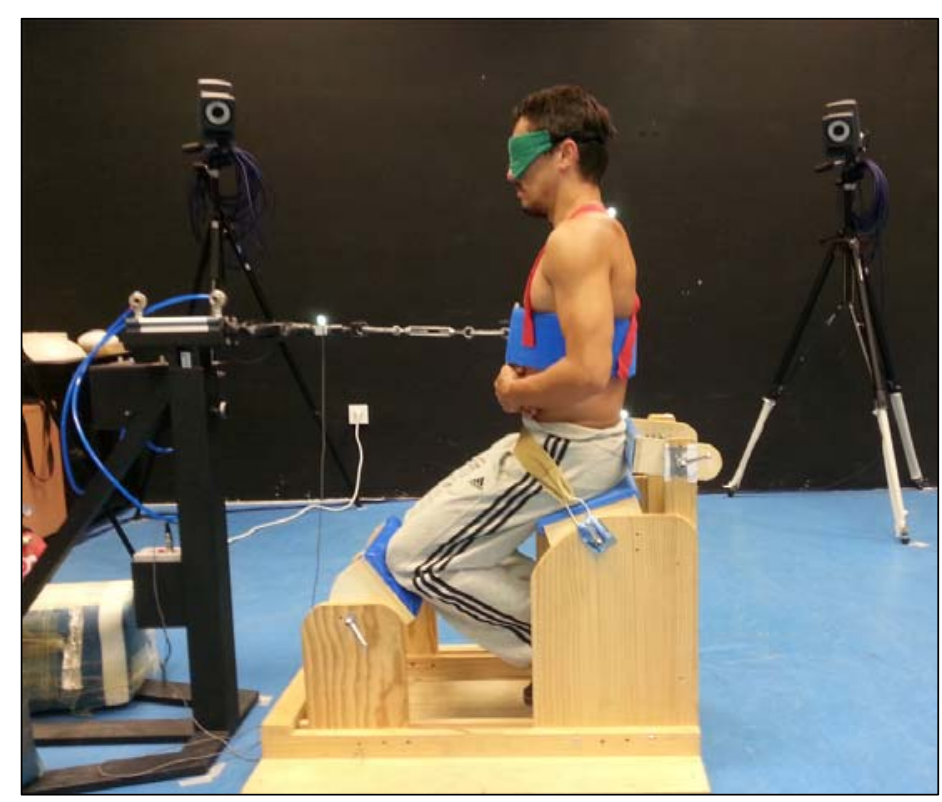

La respuesta cinemática del tronco ante las cargas súbitas se registró a $200 \mathrm{~Hz}$ con un sistema de análisis de movimiento en 3D formado por siete cámaras T10 Vicon (Vicon MX system, Oxford, UK). Antes de iniciar el test se colocaron marcadores reflectantes sobre las apófisis espinosasde L5 y C7 y sobre el arnés, a $2 \mathrm{~cm}$ aproximadamente a la derecha del centro de masas de la parte superior del cuerpo. Asimismo, también se colocó un marcador sobre la célula de carga con el fin de conocer el inicio de la perturbación. Antes de la aplicación de las cargas súbitas, se realizaron tres capturas estáticas en los diferentes sentidos de carga (anterior, posterior y lateral hacia la izquierda) para la calibración del sistema de análisis del movimiento. Los datos fueron procesados utilizando el software Nexus 1.8.2 (Vicon MX system, Oxford, UK).

\subsection{2.- Test de control postural del tronco en sedestación}

La prueba consistió en la realización de 10 tareas en sedestación de diferente dificultad, cinco realizadas sobre un asiento estable y otras cinco sobre un asiento inestable. El asiento estable era una estructura de madera con un reposapiés regulable en altura para facilitar la colocación de los miembros inferiores (Fig. 2A). La altura del reposapiés se ajustó a las características de cada participante para mantener una flexión de rodilla de $90^{\circ}$. Con el fin de evitar movimientos de la parte inferior del cuerpo, se fijaron las piernas a la silla mediante cinchas colocadas sobre los tobillos y la parte superior de los muslos, evitando de este modo cualquier movimiento de la parte inferior del cuerpo. Para la ejecución de las pruebas sobre asiento inestable, se utilizó cinta de velcro ${ }^{\circledR}$ para colocar una hemisfera de resina de poliéster (radio de la hemisfera: $35 \mathrm{~cm}$, altura del 
asiento con respecto al punto más bajo en la hemisfera: $12 \mathrm{~cm}$ ) (Fig. 2B) en la parte inferior del asiento estable, convirtiéndolo en una estructura inestable con tres grados de libertad de rotación.

Dependiendo de la tarea a realizar, el asiento estable o inestable era situado sobre una plataforma de fuerzas (Kistler, Switzerland, Model 9286AA), que registraba las fuerzas de reacción y el desplazamiento del centro de presiones ( $\mathrm{CoP}$ ) durante las pruebas. La plataforma de fuerzas se encontraba a $0.9 \mathrm{~m}$ de altura respecto al suelo, encajada en el tablero de una mesa rígida y estable (Fig. 2). La mesa tenía una barandilla de seguridad acolchada, que se colocaba alrededor de los participantes para que éstos no se cayeran y se sintieran seguros durante las tareas más difíciles. La plataforma de fuerzas fue calibrada antes de cada prueba y se utilizó una frecuencia de muestreo de $1000 \mathrm{~Hz}$.

Figura 2. Test de control postural del tronco en sedestación: A) vista lateral de un participante durante la ejecución de una prueba sobre asiento estable; B) vista lateral de un participante durante la ejecución de una prueba sobre asiento inestable.

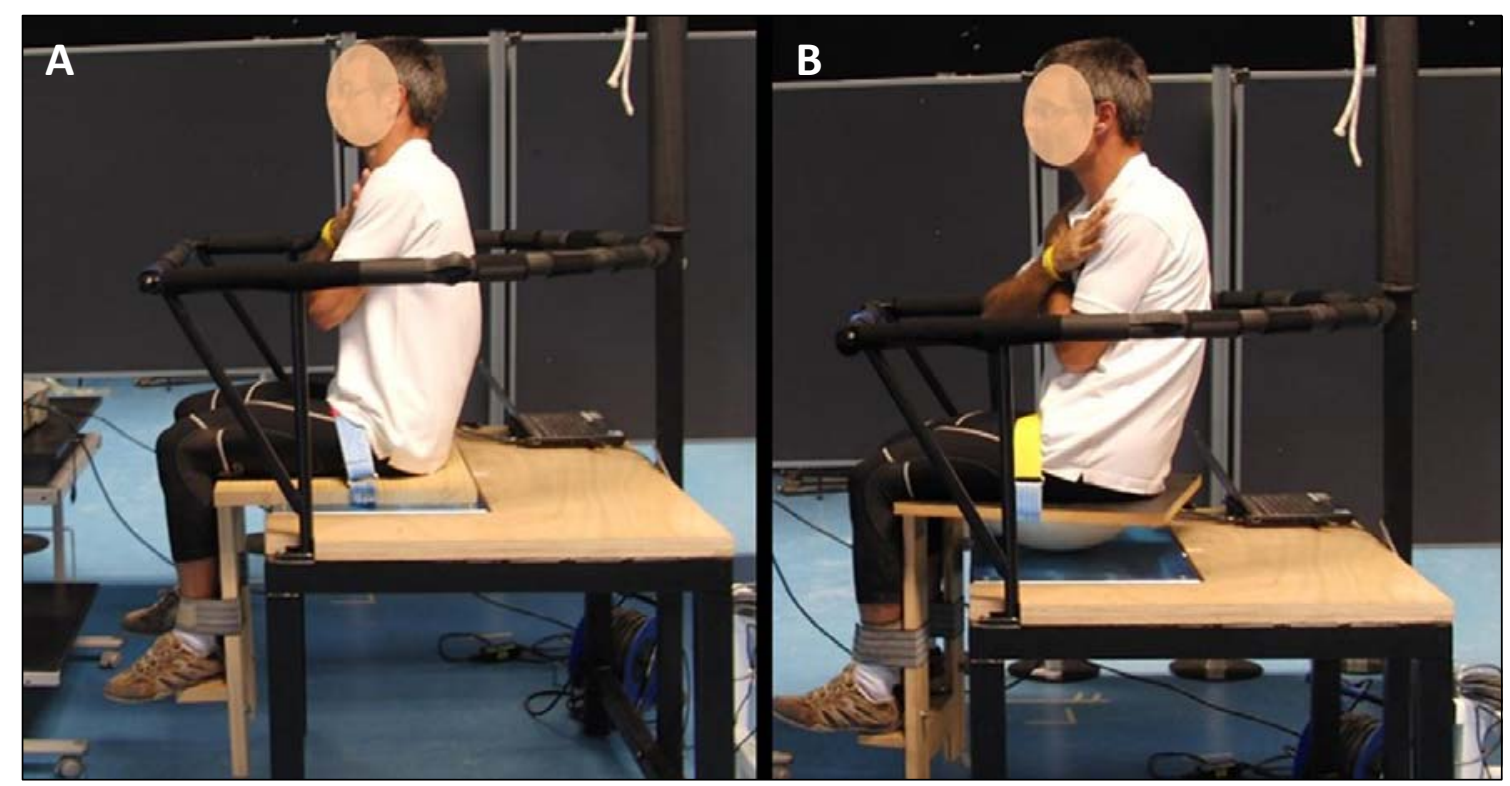

Los investigadores desarrollaron un software para proporcionar a los participantes información visual del desplazamiento de su CoP (coordenadas X e Y) en tiempo real (Fig. 3). En la imagen del software, el CoP estaba representado por un punto de color amarillo. Además, en ocho de las tareas a realizar se mostró un punto criterio de color rojo con el fin de evaluar la capacidad de los participantes para ajustar la posición de su CoP a dicho punto en distintas posiciones y trayectorias. La imagen del software fue proyectada sobre una pantalla cuyo centro estaba situado a $3.5 \mathrm{~m}$ frente al participante y a una altura de $1.1 \mathrm{~m}$. Con el objeto de facilitar la atención de los participantes sobre la información presentada en el software, el test se realizó en una sala sin ruidos, con buena iluminación y acceso restringido.

Durante el desarrollo del test, los participantes realizaron cinco pruebas sobre el asiento estable y otras cinco sobre el inestable. Dos de las cinco tareas realizadas sobre cada asiento eran estáticas y las otras tres dinámicas. Una de las dos tareas estáticas se realizó sin feedback visual, pidiendo a los participantes que se quedaran lo más quietos posible en la posición donde se encontraran más cómodos. La segunda tarea estática se llevó a cabo con feedback visual, indicando a los participantes que colocaran su CoP lo más cerca posible del punto criterio (situado en el centro de la imagen del software). En las prueba dinámicas se solicitó a los participantes que ajustaran la posición de su CoP lo más cerca posible del punto criterio (Fig. 3), que en este tipo de tareas se movía a lo largo de tres posibles trayectorias: anterior-posterior, medial-lateral y circular. Como resultado, durante el test se evaluaron 10 condiciones diferentes: prueba estática en asiento estable sin feedback; prueba estática en asiento estable con feedback; prueba estática en asiento inestable sin feedback; prueba estática en asiento inestable con feedback; prueba dinámica en asiento estable con desplazamiento medial-lateral y feedback; prueba dinámica en asiento estable 
con desplazamiento anterior-posterior y feedback; prueba dinámica en asiento estable con desplazamiento circular y feedback; prueba dinámica en asiento inestable con desplazamiento medial-lateral y feedback; prueba dinámica en asiento inestable con desplazamiento anteriorposterior y feedback; prueba dinámica en asiento inestable con desplazamiento circular y feedback.

Figura 3. Imagen del software proyectado frente al participante para proporcionar información visual del desplazamiento del centro de presiones (punto amarillo) y para retar la capacidad de éste de ajustar la posición de su CoP a un punto criterio (punto rojo) que permanecía estático o se desplazaba describiendo diferentes trayectorias. En esta imagen el participante intenta seguir el punto rojo a lo largo de una trayectoria circular. Aunque la trayectoria se presenta en la figura mediante una línea roja para que el lector la identifique claramente, no fue presentada durante el estudio.

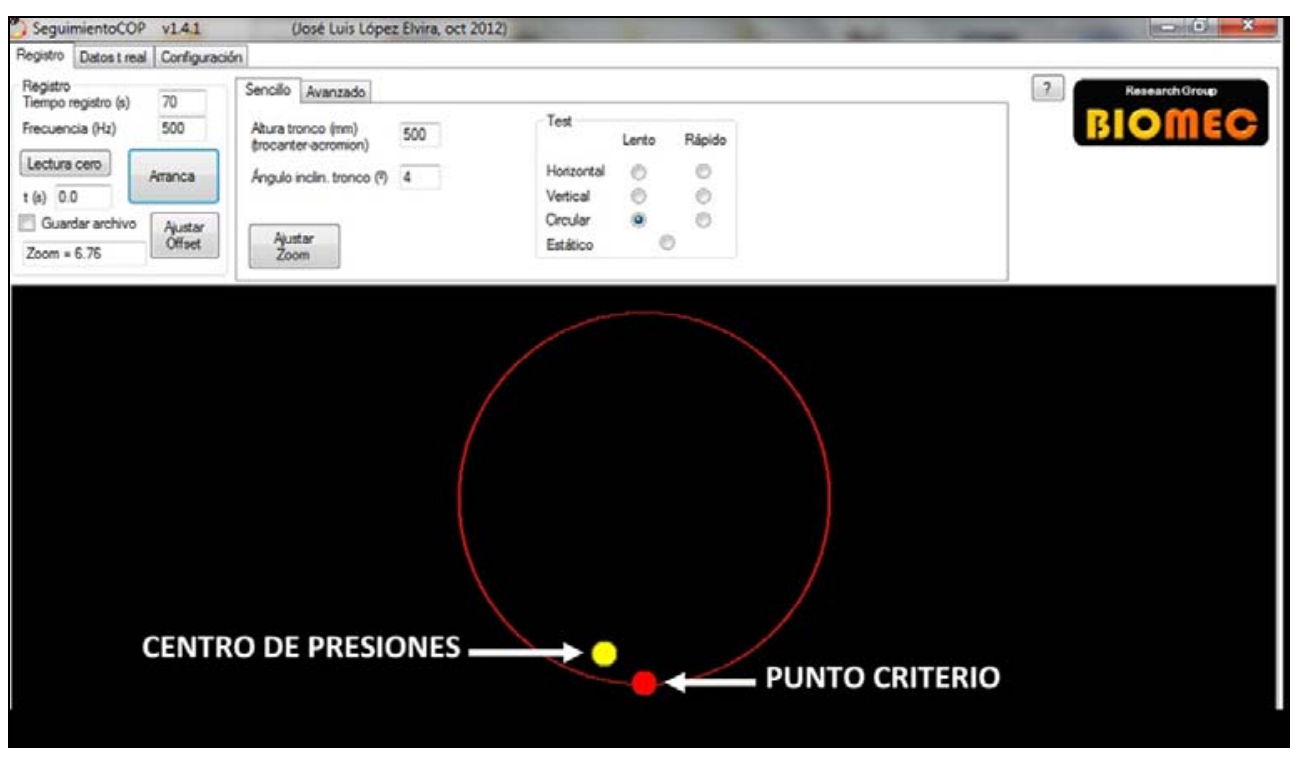

Los participantes realizaron cada tarea con los brazos cruzados sobre el pecho (Fig. 2). Todos ellos fueron capaces de mantener la posición de equilibrio sin apoyarse en la barandilla de seguridad a lo largo de las diferentes pruebas. La postura de la columna no fue controlada durante los registros. La duración de cada tarea fue de $70 \mathrm{~s}$ y el período de descanso entre las pruebas de 1 min. Cada tarea fue repetida dos veces y se analizó la segunda. Las tareas fueron contrabalanceadas para reducir un posible efecto de aprendizaje o de fatiga. Ninguno de los participantes había realizado previamente un test de estas características, por lo que el nivel de familiarización inicial con el test era el mismo para todos los deportistas.

\subsubsection{Test isocinético de fuerza y resistencia de los músculos del tronco}

Antes de comenzar el test, los participantes se sentaron sobre un módulo de tronco articulado (Dual Position Back Ex/Flex Attachment, Biodex Corporation, Shirley, NY, EE.UU.), acoplado al eje de rotación del dinamómetro isocinético Biodex® (Fig. 4). Con la ayuda de los investigadores, los participantes se colocaron en una posición inicial o de referencia donde la espalda se encontraba en posición vertical, las caderas y las rodillas flexionadas a $90^{\circ}$, los muslos paralelos al suelo y el eje de rotación del dinamómetro alineado con la línea imaginaria que une las crestas iliacas antero-superiores (Grabiner et al., 1990). Para fijar esta posición y garantizar la reproducibilidad del protocolo, se colocaron almohadillas ajustables detrás de la cabeza, el sacro y la parte superior del tronco, así como delante de la parte anterior de la tibia. Además, se colocaron unas correas inextensibles de velcro ${ }^{\circledR}$ en la parte superior del tronco (cruzadas sobre el pecho), en los muslos y en la pelvis. Como se puede ver en la figura 4, el rango de movimiento sagital del tronco se limitó a $50^{\circ}$ o de flexo-extensión, es decir, desde $30^{\circ}$ de flexión del tronco $\left(-30^{\circ}\right)$ a $20^{\circ}$ de extensión del tronco $\left(20^{\circ}\right)$, tomando como referencia la posición inicial $\left(0^{\circ}\right)$ descrita anteriormente. De acuerdo con las contribuciones de Grabiner et al. (1990), rangos de movimiento del tronco no 
superiores a $50^{\circ}$ aíslan el movimiento del raquis lumbar, evitando la flexión-extensión de la cadera. El movimiento en esta articulación se limitó también gracias a la ubicación del eje de rotación del dinamómetro a nivel de la cresta ilíaca anterior-superior y a la fijación de la pelvis mediante algunas de las almohadillas y correas comentadas anteriormente.

Figura 4. Participante realizando un esfuerzo máximo de flexión-extensión del tronco en el dinamómetro isocinético a través de un rango movimiento de 50ㅜ - $-30^{\circ}$ ) flexión del tronco; $0^{\circ}$ ) posición inicial o de referencia; $20^{\circ}$ ) extensión del tronco.
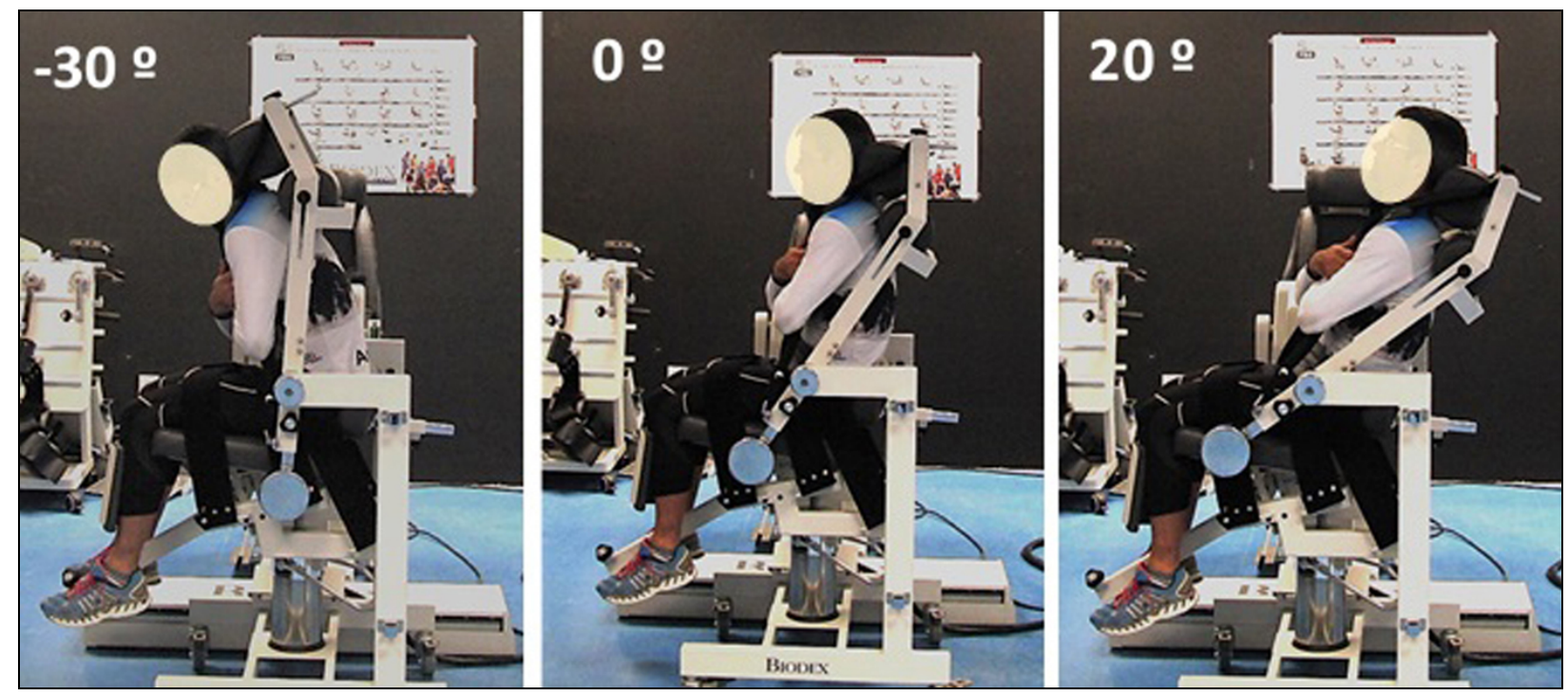

El test isocinético consistió en cuatro series de 15 repeticiones máximas y consecutivas de flexión y extensión del tronco a $120^{\circ}$ /s y en contracción concéntrica. Se eligió esta velocidad de flexo-extensión porque se considera segura para el raquis lumbar (Watkins y Harris, 1983) y fiable para medir el trabajo mecánico (Langrana et al., 1984). En cada serie, el movimiento comenzó en el sentido de la flexión. El descanso entre series fue de $1 \mathrm{~min}$. Los participantes fueron instruidos para mantener las manos y los brazos cruzados sobre el pecho durante toda la prueba y animados enérgicamente con el fin de generaran la máxima fuerza posible en cada repetición.

\subsection{Análisis de datos}

\subsubsection{Rigidez y desplazamiento del tronco ante cargas súbitas}

Para evaluar la respuesta del tronco ante las cargas aplicadas en las diferentes direcciones y sentidos, el sistema de análisis del movimiento en 3D midió el máximo desplazamiento angular del tronco $(\theta)$ en los primeros 150 ms después de aplicar las perturbaciones. Asimismo, con el objeto de analizar la respuesta del tronco en función del momento de fuerza aplicado (M), se calculó una medida básica de rigidez $(K)$ a partir de la siguiente ecuación: $K=M / \theta$.

Los momentos de fuerza aplicados de forma súbita (momento de flexión, momento de extensión y momento de inclinación lateral del tronco) se calcularon como el producto del brazo de la fuerza (distancia entre el punto de aplicación de la fuerza y L5) por la integral de las fuerzas registradas por la célula de carga durante los primeros $150 \mathrm{~ms}$ tras el inicio de la perturbación.

2.3.2. Distancia resultante del desplazamiento del CoP durante el test de control postural del tronco en sedestación

Dado que durante el test los participantes sólo estaban alineados con los ejes de la plataforma de fuerzas, se utilizó el promedio de la distancia resultante del desplazamiento del CoP como medida global para cuantificar el rendimiento (Prieto et al., 1996). Para determinar la distancia resultante en las pruebas con feedback visual, se calculó en cada instante el vector 
distancia (mm) entre la posición del CoPy la posición del punto criterio (Hancock et al., 1995; Prieto et al., 1996). Asimismo, para determinar la distancia resultante en las pruebas sin feedback visual, se calculó en cada instante el vector distancia ( $\mathrm{mm}$ ) entre la posición del CoP y el punto medio del desplazamiento realizado por el CoP durante toda la prueba (Hancock et al., 1995; Prieto et al., 1996).

\subsubsection{Fuerza y resistencia en el test isocinético de flexo-extensión del tronco}

Con el objeto de evaluar la fuerza de los músculos flexores y extensores del tronco, se calculó el momento máximo de fuerza y el trabajo mecánico total desarrollado durante las 15 repeticiones de cada serie, tanto en los movimientos de flexión como de extensión. Ambas variables se calcularon en valores absolutos y en valores relativos a la masa de los participantes. Basándose en el estudio de Mayer et al. (1995), la ratio final de fatiga, entendida como la relación entre el valor más alto del trabajo registrado en cualquiera de las repeticiones de la prueba y la media del trabajo realizado en las últimas tres repeticiones del test, fue utilizada para evaluar la resistencia muscular durante los esfuerzos de flexión y extensión del tronco.

\subsection{Análisis estadístico}

Se calcularon los estadísticos descriptivos (media y desviación estándar) para cada una de las variables. La normalidad de los datos fue examinada usando la prueba estadística de Kolmogorov-Smirnov con la corrección de Lilliefors. Con el fin de analizar las diferencias entre los dos grupos en cada una de las variables se llevó a cabo una prueba T para muestras independientes. Para analizar la relación tanto de la respuesta del tronco ante las cargas súbitas, como del control postural del tronco en sedestación, con la fuerza y la resistencia de los músculos flexores y extensores del tronco, se realizó un análisis de correlación de Pearson entre todas las variables del estudio. Los análisis se realizaron con el paquete estadístico SPSS versión 18 (SPSS Inc., Chicago, IL, USA). La hipótesis nula fue rechazada al nivel de significación del $95 \%(\mathrm{p}<.05)$.

\section{3.- Resultados}

Los estadísticos descriptivos del máximo desplazamiento angular y la rigidez del tronco tras la aplicación de cargas súbitas se presentan en la tabla 1. La prueba T no mostró diferencias significativas entre los judocas de nivel nacional e internacional en ninguna de las variables de esta prueba, mostrando ambos grupos resultados muy similares.

Tabla 1. Estadísticos descriptivos y diferencias entre grupos para el máximo desplazamiento angular $(\theta)$ y la rigidez del tronco $(K)$ en los primeros $150 \mathrm{~ms}$ tras aplicar la carga súbita en las diferentes direcciones y sentidos.

\begin{tabular}{lcccccc}
\hline \multirow{2}{*}{ Variable } & $\begin{array}{c}\text { Dirección } \\
\text { de la carga }\end{array}$ & $\begin{array}{c}\text { Todos } \\
\mathbf{( n = 1 3 )}\end{array}$ & $\begin{array}{c}\text { Judocas } \\
\text { internacionales (n=6) }\end{array}$ & $\begin{array}{c}\text { Judocas } \\
\text { nacionales (n=7) }\end{array}$ & t & $\boldsymbol{p}$ \\
\hline \multirow{2}{*}{$\boldsymbol{\theta}(\mathbf{o})$} & Anterior & $7.92 \pm 1.29$ & $7.85 \pm 0.77$ & $8.03 \pm 1.68$ & 0.337 & .742 \\
& Lateral & $4.23 \pm 1.45$ & $4.31 \pm 1.51$ & $4.17 \pm 1.51$ & -0.871 & .402 \\
& Posterior & $14.23 \pm 2.55$ & $13.67 \pm 2.75$ & $14.71 \pm 2.48$ & -0.167 & .870 \\
\hline \multirow{3}{*}{$\boldsymbol{K}(\mathbf{N m} / \mathbf{o})$} & Anterior & $1.90 \pm 0.38$ & $2.00 \pm 0.38$ & $1.81 \pm 0.40$ & 0.066 & .949 \\
& Lateral & $4.55 \pm 1.96$ & $4.51 \pm 2.11$ & $4.58 \pm 1.99$ & 0.716 & .489 \\
& Posterior & $1.16 \pm 0.33$ & $1.20 \pm 0.37$ & $1.12 \pm 0.32$ & -0.405 & .693 \\
\hline
\end{tabular}

* diferencias significativas $\mathrm{p}<.05$.

Los estadísticos descriptivos de la distancia resultante del desplazamiento del CoP en las diferentes tareas del test de control postural del tronco en sedestación se presentan en la tabla 2. En la mayoría de las pruebas analizadas, se observó menor distancia resultante en el desplazamiento del CoP en los judocas de nivel internacional, en comparación con los de nivel nacional, lo que refleja un mayor rendimiento en el test. Según la prueba T, las diferencias entre los 
grupos fueron estadísticamente significativas en la tarea más compleja, es decir, en la prueba dinámica en asiento inestable con desplazamiento circular y feedback $(\mathrm{t}=2.589 ; \mathrm{p}=.025)$.

Tabla 2. Estadísticos descriptivos y diferencias entre grupos para la distancia resultante del desplazamiento del centro de presiones en las diferentes tareas del test de control postural del tronco en sedestación.

\begin{tabular}{lcccccc}
\hline Variable & Tarea & $\begin{array}{c}\text { Todos } \\
(\mathbf{n = 1 3 )}\end{array}$ & $\begin{array}{c}\text { Judocas internacionales } \\
(\mathbf{n = 6 )}\end{array}$ & $\begin{array}{c}\text { Judocas nacionales } \\
(\mathbf{n = 7})\end{array}$ & $\mathbf{t}$ & $\boldsymbol{p}$ \\
\hline & EESF & $0.94 \pm 0.38$ & $0.86 \pm 0.38$ & $1.00 \pm 0.39$ & 0.634 & .539 \\
& EECF & $0.87 \pm 0.49$ & $0.76 \pm 0.26$ & $0.97 \pm 0.64$ & 0.749 & .470 \\
& EISF & $5.92 \pm 1.67$ & $5.66 \pm 1.50$ & $6.15 \pm 1.89$ & 0.506 & .623 \\
Distancia & EICF & $5.34 \pm 1.72$ & $4.54 \pm 1.26$ & $6.02 \pm 1.85$ & 1.643 & .129 \\
resultante & DEML & $2.28 \pm 0.44$ & $2.33 \pm 0,57$ & $2,24 \pm 0,34$ & -0.362 & .724 \\
(mm) & DEAP & $2.23 \pm 0.44$ & $2.14 \pm 0.49$ & $2.31 \pm 0.42$ & 0.675 & .513 \\
& DECI & $3.35 \pm 0.93$ & $3.37 \pm 0,75$ & $3.33 \pm 0.93$ & -0.073 & .943 \\
& DIML & $7.14 \pm 1.93$ & $6.39 \pm 1.24$ & $7.79 \pm 2.26$ & 1.344 & .206 \\
& DIAP & $6.70 \pm 1.08$ & $6.67 \pm 1.55$ & $6.73 \pm 0.55$ & 0.096 & .925 \\
& DICI & $8.04 \pm 1.63$ & $7.00 \pm 1.19$ & $8.93 \pm 1.45$ & 2.589 & $.025^{*}$ \\
\hline
\end{tabular}

Prueba estática en asiento estable sin feedback (EESF); prueba estática en asiento estable con feedback (EECF); prueba estática en asiento inestable sin feedback (EISF); prueba estática en asiento inestable con feedback (EICF); prueba dinámica en asiento estable con desplazamiento medial-lateral y feedback (DEML); prueba dinámica en asiento estable con desplazamiento anterior-posterior y feedback (DEAP); prueba dinámica en asiento estable con desplazamiento circular y feedback (DECI); prueba dinámica en asiento inestable con desplazamiento medial-lateral y feedback (DIML); prueba dinámica en asiento inestable con desplazamiento anterior-posterior y feedback (DIAP); prueba dinámica en asiento inestable con desplazamiento circular y feedback (DICI); * diferencias significativas $\mathrm{p}<.05$.

Los estadísticos descriptivos de las variables del test isocinético de fuerza y resistencia de los músculos del tronco se presentan en la tabla 3. Según la prueba T, los judocas de nivel internacional mostraron mayor momento de fuerza extensora que los judocas de nivel nacional, tanto en valores absolutos $(t=-2.215 ; \mathrm{p}=.049)$ como relativos $(\mathrm{t}=-2.969 ; \mathrm{p}=.013)$. No se encontraron diferencias estadísticas entre grupos en el resto de variables de fuerza y resistencia muscular.

Tabla 3. Estadísticos descriptivos y diferencias entre grupos en el test isocinético de fuerza y resistencia de la musculatura del tronco.

\begin{tabular}{|c|c|c|c|c|c|c|}
\hline Variables & $\begin{array}{l}\text { Movimiento } \\
\text { del tronco }\end{array}$ & Todos & $\begin{array}{c}\text { Judocas } \\
\text { Internacionales } \\
(n=6)\end{array}$ & $\begin{array}{c}\text { Judocas } \\
\text { Nacionales } \\
(n=7)\end{array}$ & $\mathbf{t}$ & $p$ \\
\hline \multirow{2}{*}{ MM (Nm) } & Extensión & $445.08 \pm 74.82$ & $488.21 \pm 69.60$ & $408.11 \pm 60.88$ & -2.215 & $.049^{*}$ \\
\hline & & $224.78 \pm 22.43$ & $221.75 \pm 28.98$ & $227.38 \pm 17.01$ & 0.436 & .671 \\
\hline \multirow{2}{*}{$\begin{array}{l}\text { MMR } \\
\text { (Nm/kg) }\end{array}$} & Extensión & $6.34 \pm 1.02$ & $7.05 \pm 0.87$ & $5.74 \pm 0.72$ & -2.969 & $.013^{*}$ \\
\hline & Flexión & $3.20 \pm 0.26$ & $3.19 \pm 0.26$ & $3.21 \pm 0.29$ & 0.094 & .927 \\
\hline \multirow{2}{*}{ TT (J) } & Extensión & $2617.04 \pm 516.73$ & $2808.06 \pm 520.52$ & $24530 \pm 490.38$ & -1.264 & .232 \\
\hline & Flexi & $1119.65 \pm 302.90$ & $983.59 \pm 392.54$ & $1236.27 \pm 143.93$ & 1.592 & .140 \\
\hline \multirow{2}{*}{$\begin{array}{l}\text { TTR } \\
\text { (J/kg) }\end{array}$} & Extensión & $37.19 \pm 6.23$ & $40.36 \pm 5.10$ & $34.48 \pm 6.11$ & -1.858 & .090 \\
\hline & Flexión & $15.87 \pm 4.18$ & $13.97 \pm 5.07$ & $17.50 \pm 2.61$ & 1.614 & .135 \\
\hline \multirow{2}{*}{ RFF (\%) } & Extensión & $72.99 \pm 9.36$ & $71.76 \pm 11.02$ & $74.03 \pm 8.45$ & 0.420 & .682 \\
\hline & Flexión & $65.32 \pm 8.47$ & $67.77 \pm 7.76$ & $64.94 \pm 9.63$ & -0.171 & .868 \\
\hline
\end{tabular}

Máximo momento de fuerza (MM); máximo momento de fuerza relativo (MMR); trabajo total (TT); trabajo total relativo (TTR); ratio final de fatiga (RFF); * diferencias significativas $\mathrm{p}<.05$.

Los resultados del análisis de las correlaciones se muestran en las tablas 4 y 5 . Las variables de fuerza y resistencia del test isocinético de flexo-extensión del tronco sólo mostraron 5 correlaciones positivas significativas con variables del test de control postural sedente y 2 correlaciones positivas significativas con variables del test de aplicación de cargas súbitas. 
Tabla 4. Correlaciones entre las variables de fuerza y resistencia del test isocinético de flexo-extensión del tronco y la distancia resultante del desplazamiento del centro de presiones en las diferentes tareas del test de control postural sedente.

\begin{tabular}{|c|c|c|c|c|c|c|c|c|c|c|c|}
\hline Variable & $\begin{array}{l}\text { Movimiento } \\
\text { del tronco }\end{array}$ & EESF & EECF & EISF & EICF & DEML & DEAP & DECI & DIML & DIAP & DICI \\
\hline \multirow{2}{*}{ MM } & Extensión & .149 & 7 & .153 & .106 & 0 & 430 & 2 & -.260 & .119 & -.195 \\
\hline & Flexión & .368 & .217 & .525 & .358 & -.161 & -.095 & .248 & .146 & .048 & .167 \\
\hline \multirow{2}{*}{ MMR } & Extensión & .107 & -.350 & .125 & .000 & & -.351 & -.204 & -.237 & .213 & -.204 \\
\hline & Flexión & .432 & .045 & $.636 *$ & .279 & -.146 & .108 & .226 & .298 & .270 & .252 \\
\hline \multirow{2}{*}{ TT } & Extensión & .269 & -.096 & .140 & .198 & .069 & -.258 & -.076 & -.261 & .216 & .025 \\
\hline & Flexión & .538 & .194 & $.592 *$ & .353 & .194 & .293 & .227 & .376 & .096 & .328 \\
\hline \multirow{2}{*}{ TTR } & Extensión & .288 & -.222 & .143 & .133 & .110 & -.180 & -.127 & -.267 & .361 & .049 \\
\hline & Flexión & $.581 *$ & .143 & $.646 *$ & .334 & .408 & .408 & .229 & .448 & .174 & .386 \\
\hline \multirow{2}{*}{ RFF } & Extensión & -.070 & -.214 & .114 & .313 & .142 & -.156 & -.236 & .250 & -.347 & -.019 \\
\hline & Flexión & .405 & .096 & .267 & .068 & .436 & $.654^{*}$ & -.021 & -.024 & .280 & .223 \\
\hline
\end{tabular}

Máximo momento de fuerza (MM); máximo momento de fuerza relativo (MMR); trabajo total (TT); trabajo total relativo (TTR); ratio final de fatiga (RFF); prueba estática en asiento estable sin feedback (EESF); prueba estática en asiento estable con feedback (EECF); prueba estática en asiento inestable sin feedback (EISF); prueba estática en asiento inestable con feedback (EICF); prueba dinámica en asiento estable con desplazamiento medial-lateral y feedback (DEML); prueba dinámica en asiento estable con desplazamiento anterior-posterior y feedback (DEAP); prueba dinámica en asiento estable con desplazamiento circular y feedback (DECI); prueba dinámica en asiento inestable con desplazamiento medial-lateral y feedback (DIML); prueba dinámica en asiento inestable con desplazamiento anterior-posterior y feedback (DIAP); prueba dinámica en asiento inestable con desplazamiento circular y feedback (DICI); * Significación p < .05.

Tabla 5. Correlaciones entre las variables de fuerza y resistencia del test isocinético de flexo-extensión del tronco y las variables del test de cargas súbitas aplicadas en diversas direcciones.

\begin{tabular}{|c|c|c|c|c|c|c|c|}
\hline Variable & $\begin{array}{l}\text { Movimiento } \\
\text { del tronco }\end{array}$ & $\theta$ anterior & Kanterior & $\theta$ lateral & Klateral & Oposterior & Kposterior \\
\hline \multirow{2}{*}{ MM } & Extensión & -.280 & .435 & .069 & .120 & -.374 & .284 \\
\hline & Flexión & -.198 & .492 & -.174 & .367 & -.541 & $.619 *$ \\
\hline \multirow{2}{*}{ MMR } & Extensión & -.130 & .220 & -.025 & .170 & -.163 & .133 \\
\hline & Flexión & .096 & .133 & -.371 & .519 & -.286 & .500 \\
\hline \multirow{2}{*}{ TT } & Extensión & -.454 & $.587^{*}$ & .061 & .170 & -.240 & .133 \\
\hline & Flexión & .078 & .057 & .162 & .000 & -.457 & .515 \\
\hline \multirow{2}{*}{ TTR } & Extensión & -.359 & .436 & -.020 & .242 & -.042 & -.012 \\
\hline & Flexión & .207 & -.105 & .106 & .044 & -.346 & .458 \\
\hline \multirow{2}{*}{ RFF } & Extensión & -.054 & -.105 & .151 & -.350 & .064 & -.317 \\
\hline & Flexión & -.323 & .129 & .045 & .022 & .115 & -.121 \\
\hline
\end{tabular}

Máximo momento de fuerza (MM); máximo momento de fuerza relativo (MMR); trabajo total (TT); trabajo total relativo (TTR); ratio final de fatiga (RFF); desplazamiento angular $(\theta)$; rigidez $(K) ; *$ Significación p< .05 .

\section{4.- Discusión}

Teniendo en cuenta los resultados de estudios biomecánicos previos (Elvira et al., en prensa; Juan-Recio et al., 2013; Kort y Hendriks, 1992; López-Valenciano et al., 2013), el control de la estabilidad postural y la fuerza de la musculatura del tronco son cualidades que parecen caracterizar al judoca de competición y lo diferencian de otros deportistas. En este estudio se analizó si la estabilidad y la condición muscular del tronco permiten diferenciar también entre judocas de nivel nacional e internacional. Asimismo, se realizó un análisis correlacional para valorar la relación entre los resultados obtenidos por los judocas en los diferentes test.

En judo, los competidores tienen que tener un buen control postural, ya que las técnicas de este deporte se basan principalmente en desplazamientos constantes y perturbaciones externas inesperadas realizadas por sus oponentes con el objetivo de desequilibrar y proyectar durante el combate (Perrot et al., 1998; Perrin et al., 2002). Sin embargo, los resultados en el test de aplicación de cargas súbitas no mostraron diferencias significativas entre los judocas de las distintas 
categorías (tabla 1). El test de cargas súbitas mide el desplazamiento y la rigidez del tronco durante los $150 \mathrm{~ms}$ posteriores a la aplicación de las perturbaciones, lo que representa una respuesta involuntaria ante este tipo de fuerzas, especialmente, la respuesta de las estructuras pasivas y la respuesta muscular refleja del tronco (Vera-Garcia et al., 2006). Aunque el estudio de LopezValenciano et al. (2013) sí encontró diferencias en la respuesta involuntaria del tronco ante cargas súbitas laterales entre judocas de competición e individuos que practicaban otros deportes de forma recreativa, nuestros datos no permiten diferenciar diversos niveles de rendimiento en judocas de competición. De forma similar, Yoshitomi et al. (2006) tampoco encontraron diferencias en la respuesta refleja, ni en la amplitud ni en la velocidad del desplazamiento del CoP entre judocas de diferente nivel (cinturón verde vs. cinturón marrón), utilizando un protocolo de perturbaciones repentinas e inesperadas para valorar el control del equilibrio corporal mediante la aplicación de cargas en dirección antero-posterior en bipedestación. Sin embargo, estos mismos autores sí encontraron diferencias significativas entre los judocas de mayor nivel (cinturón marrón) y deportistas recreacionales en la velocidad de desplazamiento del CoP, parámetro ampliamente utilizado para evaluar el control postural (Duarte, 2000).

En referencia al test de control postural del tronco en sedestación, los judocas de categoría internacional obtuvieron un mayor rendimiento que los judocas de categoría nacional en la mayoría de pruebas analizadas en el test, siendo significativa la diferencia únicamente en la tarea más compleja (tabla 2). Diversos autores (Perrin et al., 2002; Yoshitomi et al., 2006) señalan que el entrenamiento reta al sistema nervioso central a controlar la posición del centro de gravedad del cuerpo favoreciendo la organización de patrones posturales en tareas de equilibrio en función de la información sensorial disponible y los condicionantes biomecánicos (Allum et al., 1998; Dietz et al., 1993). Estos resultados, unidos a la ausencia de diferencias entre los judocas al analizar la respuesta involuntaria del tronco ante las cargas súbitas, podrían sugerir que la diferencia en el control del tronco entre judocas de competición se manifiesta en condiciones dinámicas donde el control voluntario es un factor determinante. Esto estaría en relación con el hecho de que durante un combate, el judoca se encuentra en un estado continuo de intercambio de fuerzas con el oponente produciéndose numerosos desequilibrios (Perrin et al., 2002; Yoshitomi et al. 2006), lo cual podría requerir continuos ajustes por parte del judoca. De cualquier forma, futuros estudios deben profundizar en el análisis de la estabilidad del tronco de los judocas en diferentes condiciones (estáticas, dinámicas, en sedestación, en bipedestación, etc.), ya que la estabilidad es dependiente del contexto (Reeves et al., 2007) y por tanto, como se ha observado en este estudio, se puede manifestar de forma distinta en función de las características del test utilizado. Así, es posible que si en el test de aplicación de cargas súbitas se hubiera analizado la respuesta voluntaria ante las cargas, en lugar de la involuntaria, los resultados hubieran sido diferentes.

En cuanto a los resultados obtenidos en el test isocinético de fuerza y resistencia de los músculos del tronco, los judocas de categoría internacional mostraron mayor momento de fuerza extensora que los judocas de nivel nacional, tanto en valores absolutos como relativos. Según estos resultados, la fuerza extensora de la musculatura del tronco es una variable que permite diferenciar entre judocas de competición de diferente nivel de rendimiento. En este sentido, Kort y Hendricks (1992), encontraron valores superiores en la fuerza de la musculatura extensora del tronco respecto a la fuerza de la musculatura flexora, tanto para el máximo momento de fuerza como para el trabajo total en judocas de nivel nacional e internacional. Esto puede ser debido a la importancia de los movimientos de extensión del tronco en las acciones de ataque y defensa durante los entrenamientos y/o competiciones de judo. En cualquier caso, debido a las adaptaciones producidas por la práctica de judo en el desarrollo de la fuerza y resistencia de los músculos del tronco, los judocas se caracterizan por tener grandes niveles de fuerza en comparación con otros deportistas (Elvira et al., en prensa, Juan-Recio et al., 2013; Kort y Hendriks, 1992), si bien no existen evidencias suficientes que permitan determinar en qué medida el desarrollo de estas capacidades está relacionado con un mayor rendimiento deportivo en este u otros deportes (Reed et al., 2012).

Aunque la fuerza de la musculatura extensora y el control de la estabilidad postural del tronco parecen influir en el rendimiento del judoca, los datos obtenidos en este estudio indican que no existe una relación clara entre las variables de condición muscular y las variables relacionadas con la estabilidad del tronco (tablas 4 y 5). Algunos estudios proponen la utilización de test de 
resistencia o test de fuerza-potencia muscular como medidas indirectas de estabilidad del tronco (Akuthota et al., 2008; Cowley y Swensen, 2008; Cowley et al., 2009; Leetun et al., 2004; Nesser et al., 2008), sin embargo, según nuestros resultados, los test de condición muscular no parecen pruebas válidas para medir la estabilidad. En este sentido, aunque la resistencia y la fuerza muscular, al igual que otras variables como la edad, la altura o la patología lumbar, son variables que pueden influir en la capacidad de estabilización de las estructuras de la zona central del cuerpo, los resultados de los test de resistencia o fuerza muscular no son medidas de estabilidad en sí mismas (van Dieën, 2012).

Debemos resaltar que debido a las características de la muestra y su difícil accesibilidad, el tamaño de la misma fue pequeño, por lo que los resultados de este estudio deben analizarse con cautela. Es necesario realizar nuevos estudios biomecánicos con mayor número de participantes y niveles de rendimiento, así como estudios experimentales que nos permitan conocer realmente el efecto del desarrollo de las funciones de los músculos del tronco sobre el rendimiento de los judocas. Además, en relación con la ratio final de fatiga, variable utilizada en nuestro estudio para medir la resistencia de la musculatura del tronco, debemos indicar que esta podría estar influenciada por valores particularmente elevados de trabajo mecánico máximo, como sugieren los datos obtenidos por Girard et al. (2011) en un estudio sobre la fatiga en un test de sprints repetidos. Futuros estudios deberían tener en cuenta esta circunstancia y desarrollar otras variables de resistencia para analizar su relación con la estabilidad del tronco. A pesar de estas limitaciones, el presente estudio aporta información relevante y novedosa, especialmente por la complejidad de las metodologías utilizadas y la dificultad para acceder a deportistas de nivel internacional.

\section{5.- Conclusiones}

La condición muscular y la estabilidad del tronco son cualidades a tener en cuenta en la preparación física de los judocas, ya que según nuestros resultados, los judocas de categoría internacional mostraron mayor control postural del tronco y mayor fuerza isocinética de la musculatura extensora que los judocas de categoría nacional. Por otro lado, el análisis correlacional no encontró una relación clara entre las variables de condición muscular y las variables de estabilidad del tronco, por lo que la fuerza y resistencia muscular no parecen tener un efecto importante sobre el rendimiento en los test de estabilidad utilizados en este estudio.

\section{Nota}

Una versión previa de este trabajo se presentó en el I Congreso Internacional de Judo De FrutosUMH, Alicante, 27-30 de junio de 2013.

\section{Agradecimientos}

Los autores agradecen la colaboración de los participantes en el estudio, el cual se llevó a cabo gracias a la ayuda económica recibida por el Ministerio de Ciencia e Innovación (Plan Nacional de I+D+I; Ref.: DEP2010-16493) y la Escuela de Estudios Universitarios Real Madrid - Universidad Europea de Madrid (Ref.: UEM1.11X). Casto Juan-Recio y Alejandro López-Valenciano han participado en este estudio gracias sendas becas pre-doctorales concedidas por la Generalitat Valenciana $($ Val i $+d)$.

\section{Referencias}

Akuthota, V., Ferreiro, A., Moore, T., \& Fredericson, M. (2008). Core stability exercise principles. Current Sports Medicine Reports, 7(1), 39-44.

Allum, J.H.J., Bloem, B.R., Carpenter, M.G., Hulliger, M., \& Hadders-Algra M. (1998). Proprioceptive control of posture: a review of new concepts. Gait Posture, 8, 214-242. 
Borghuis, J., Hof, A.L., \& Lemmink, K.A. (2008). The importance of sensory-motor control in providing core stability: implications for measurement and training. Sports Medicine, 38(11), 893-916.

Burg, J.C.E., Wegen, E.E.H., Rietberg, M.B., Kwakkel, G., \& van Dieën, J.H. (2006). Postural control of the trunk during unstable sitting in Parkinson's disease. Parkinsonism \& Related Disorders, 12, 492-498.

Cowley, P.M., Fitzgerald, S., Sottung, K., \& Swensen, T. (2009). Age, weight, and the front abdominal power test as predictors of isokinetic trunk strength and work in young men and women. Journal of Strength \& Conditioning Research, 23(3), 915-925.

Cowley, P.M., \& Swensen, T.C. (2008). Development and reliability of two core stability field test. Journal of Strength \& Conditioning Research, 22(2), 619-624.

Cholewicki, J., Greene, H.S., Polzhofer, G.K., Galloway, M.T., Shah, R.A., \& Radebold, A. (2002). Neuromuscular function in athletes following recovery from a recent acute low back injury. Journal of Orthopaedic \& Sports Physical Therapy, 32(11), 568-575.

Cholewicki, J., Polzhofer, G.K., \& Radebold, A. (2000). Postural control of trunk during unstable sitting. Journal of Biomechanics, 33(12), 1733-1737.

Dietz, V., Trippel, M., Ibrahim, I.K., \& Berger, W. (1993). Human stance on a sinusoidally translating platform: balance control by feedforward and feedback mechanisms. Experimental Brain Research, 93,352-362.

Duarte, M. (2000). Análise estabilográfica da postura ereta humana quasi-estática [tese livredocência]. São Paulo: Escola de Educação Física e Esporte, Universidade de São Paulo.

Elvira, J.L.L., Barbado, D., Juan-Recio, C., García-Vaquero, M.P., López-Valenciano, A., López-Plaza, D., Montero-Carretero, C., \& Vera-Garcia, F.J. Diferencias en la estabilización del tronco sobre un asiento inestable entre piragüistas, judocas y sujetos físicamente activos. Revista Kronos (En prensa).

Girard, O., Mendez-Villanueva, A., \& Bishop, D. (2011). Repeated-Sprint Ability - Part I. Factors Contributing to Fatigue. Sports medicine, 41(8), 673-694.

Grabiner, M.D., Jeziorowski, J.J., \& Divekar, A.D. (1990). Isokinetic measurements of trunk extension and flexion performance collected with the biodex clinical data station. Journal of Orthopaedic \& Sports Physical Therapy, 11, 590-598.

Hancock, G.R., Butler, M.S., \& Fischman, M.G. (1995). On the Problem of Two-Dimensional Error Scores: Measures and Analyses of Accuracy, Bias, and Consistency. Journal of Motor Behavior, 27, 241-250.

Juan-Recio, C., Lopez-Valenciano, A., Barbado, D., Lopez-Plaza, D., Montero, C., \& Vera-Garcia, F.J. (2013). Efecto de la condición muscular en la estabilidad del tronco en judocas de élite. Trabajo presentado en el I Congreso Internacional de Judo De Frutos-UMH, Alicante.

Kibler, W.B., Press, J., \& Sciascia A. (2006). The role of core stability in athletic function. Sports Medicine, 36(3), 189-198.

Kort, H.D., \& Hendriks, E.R. (1992). A comparison of selected isokinetic trunk strength parameters of elite male judo competitors and cyclists. Journal of Orthopaedic \& Sports Physical Therapy, 16(2), 92-96.

Langrana, N.A., Lee, C.K., Alexander, H., \& Mayott, C.W. (1984). Quantitative assessment of back strength using isokinetic testing. Spine, 9, 287-290.

Lee, H., \& Granata, K.P. (2008). Process stationarity and reliability of trunk postural stability. Clinical Biomechanics, 23(6), 735-742.

Leetun, D.T., Ireland, M.L., Willson, J.D., Ballantyne, B.T., \& Davis, I.M. (2004). Core stability measures as risk factors for lower extremity injury in athletes. Medicine \& Science in Sports \& Exercise, 36(6), 926-934.

López-Valenciano, A., Juan-Recio, C., Barbado, D., López-Elvira, J.L., Montero, C., \& Vera-Garcia, F.J. (2013). Perfil de la estabilidad mecánica del tronco en judocas de elite. Trabajo presentado en el I Congreso Internacional de Judo De Frutos-UMH. Alicante.

Mayer, T., Gatchel, R., Betancur, J., \& Bovasso, E. (1995). Trunk muscle endurance measurement. Isometric contrasted to isokinetic testing in normal subjects. Spine, 20, 920-927.

McGill, S.M. (2002). Low back disorders. Evidence-based prevention and rehabilitation. Champaign, Illinois: Human Kinetics. 
Nesser, T. W., Huxel, K. C., Tincher, J. L., \& Okada, T. (2008). The relationship between core stability and performance in division I football players. Journal Strength \& Conditional Research, 22(6), 1750-1754.

Perrot, C., Deviterne, D., \& Perrin, P.H. (1998). Influence of training on postural and motor control in a combative sport. Journal of Human Movement Studies, 35, 119-135.

Perrin, P., Deviterne, D., Hugel, F., \& Perrot, C. (2002). Judo, better than dance, develops sensorimotor adaptabilities involved in balance control. Gait Posture, 15(2), 187-194.

Prieto, T.E., Myklebust, J.B., Hoffmann, R.G., Lovett, E.G., \& Myklebust, B.M. (1996). Measures of postural steadiness: differences between healthy young and elderly adults. IEEE Transactions on Biomedical Engineering, 43, 956-966.

Preuss, R. \& Fung, J. (2008). Musculature and biomechanics of the trunk in the maintenance of upright posture. Journal of Electromyography \& Kinesiology, 18(5), 815-828.

Reed, C.A., Ford, K.R., Myer, G.D., \& Hewett, T.E. (2012). The effects of isolated and integrated 'core stability' training on athletic performance measures: a systematic review. Sports Medicine, 42(8), 697-706.

Reeves, N.P., Narendra, K.S., \& Cholewicki, J. (2007). Spine stability: The six blind men and the elephant. Clinical Biomechanics, 21(5), 266-274.

Sutarno, C.G. \& McGill, S.M. (1995). Isovelocity investigation of the lengthening behaviour of the erector spinae muscles. European Journal of Applied Physiology and Occupational Physiology, 70, 146-153.

Sung, P.S., Yoon, B., \& Lee, D.C. (2010). Lumbar spine stability for subjects with and without low back pain during one-leg standing test. Spine, 35(16), E753-760.

vanDieën, J.H., Koppes L.L.J., \& Twisk, J. (2010a). Low-back pain history and postural sway in unstable sitting. Spine, 35, 812-817.

vanDieen, J.H., Koppes, L.L., \& Twisk, J.W. (2010b). Postural sway parameters in seated balancing; their reliability and relationship with balancing performance. Gait Posture, 31(1), 42-46.

vanDieën, J.H., Luger, T., \& van der, Eb. (2012). Effects of fatigue on trunk stability in elite gymnasts. European Journal of Applied Physiology, 112(4), 1307-13.

Vera-Garcia, F.J., Brown, S.H.M., Gray, J.R., \& McGill, S.M. (2006). Effects of different levels of torso coactivation on trunk muscular and kinematic responses to posteriorly applied sudden loads. Clinical Biomechanics, 21(5), 443- 455.

Vera-Garcia, F.J., Elvira, J.L.L., Brown, S.H.M., \& McGill, S.M. (2007). Effects of abdominal stabilization maneuvers on the control of spine motion and stability against sudden trunk perturbations. Journal of Electromyography and Kinesiology, 17(5), 556-567.

Watkins, M.P. \& Harris, B.A. (1983). Evaluation of isokinetic muscle performance. Clinics in Sports Medicine, 2, 37-53.

Winter, D. A. (1990). Biomechanics and Motor Control of Human Movement. $2^{\text {nd }}$ ed. Hoboken - New Jersey: Wiley.

Yoshitomi, S.K., Tanaka, C., \& Duarte, M. (2006). Postural responses to unexpected external perturbance in judoists of different ability levels. Revista Brasileira de Medicina do Esporte, 1(12), 145e-148e.

Zazulak, B., Cholewicki, J., \& Reeves, N.P. (2008). Neuromuscular control of trunk stability: clinical implications for sports injury prevention. Journal of the American Academy of Orthopaedic Surgeons, 16(9), 497-505.

Zazulak, B.T., Hewett, T.E., Reeves, N.P., Goldberg, B., \& Cholewicki, J. (2007). Deficits in neuromuscular control of the trunk predict knee injury risk: a prospective biomechanicalepidemiologic study. The American Journal of Sports Medicine, 35(7), 1123-30.

\section{Datos biográficos de los autores}

Casto Juan-Recio es Doctorando en Psicología de la Salud. Licenciado en Ciencias de la Actividad Física y el Deporte y Master en Rendimiento Deportivo y Salud, actualmente es profesor en Actividad Física en la Naturaleza en la Universidad Miguel Hernández de Elche. E-mail: cjuan@goumh.umh.es. 
David Barbado es Doctorando en Psicología de la Salud. Licenciado en Ciencias de la Actividad Física y el Deporte, Diplomado en Magisterio de Educación Física y Master en Motricidad Humana. Actualmente es profesor en Biomecánica del Movimiento Humano y Actividad Física en la Naturaleza en la Universidad Miguel Hernández de Elche. E-mail: dbarbado@umh.es.

Alejandro López-Valenciano es Doctorando en Psicología de la Salud. Licenciado en Ciencias de la Actividad Física y el Deporte y Master en Rendimiento Deportivo y Salud. Becario de investigación (Vali+d) de la Conselleria de Educación de la Generalitat Valenciana. E-mail: alejandro.lopezv@umh.es.

Diego López-Plaza es Diplomado en Magisterio de Educación Física por la Universidad de Almería y Licenciado en Ciencias de la Actividad Física y el Deporte, por la Universidad Miguel Hernández de Elche. Máster en Rendimiento Deportivo y Salud y actualmente doctorando en Psicología de la Salud en la Universidad Miguel Hernández de Elche. E-mail: dlp_arriero@hotmail.com.

Carlos Montero-Carretero es Doctor en Psicología y especialista en motivación deportiva. Licenciado en Ciencias de la Actividad Física y el Deporte, Entrenador Nacional de Judo y 5o Dan, actualmente es profesor de Deportes de Lucha en la Universidad Miguel Hernández de Elche y Director-Entrenador del De Frutos Club de Judo de Alicante. Como competidor del Equipo Nacional de Judo fue Bicampeón de España Senior, Bronce en el Campeonato del Mundo Universitario, y Bronce en Campeonato de Europa por Equipos. E-mail: cmontero@umh.es.

Francisco J. Vera-Garcia es Doctor en Ciencias de la Actividad Física y el Deporte y especialista en Biomecánica del Raquis y acondicionamiento de la musculatura del tronco (core training). Actualmente es Director del Grupo de Investigación en Biomecánica para la Salud y el Rendimiento Deportivo (BIOMEC) y Coordinador del Laboratorio de Biomecánica y Salud del Centro de Investigación del Deporte de la Universidad Miguel Hernández de Elche, así como Subdirector del Máster Universitario en Rendimiento Deportivo y Salud y Secretario del Departamento de Psicología de la Salud de la misma Universidad. E-mail: fvera@umh.es. 